\title{
Digital Image Steganography using Nine-Pixel Differencing and Modified LSB Substitution
}

\author{
Gandharba Swain \\ Department of Information Technology, GMR Institute of Technology, \\ Rajam-532127, Srikakulam, Andhra Pradesh, India; gswain1234@gmail.com
}

\begin{abstract}
To provide higher embedding capacity without sacrificing the imperceptibility, a novel steganographic technique based on nine-pixel differencing with modified Least Significant Bit (LSB) substitution is proposed. The image is divided into $3 \times 3$ non-overlapping blocks. In each block the average difference value is calculated. Based on this value the block is classified to fall into one of the four levels such as, lower, lower-middle, higher-middle, and higher. If a block belongs to lower level then 2-bit LSB substitution is used in it. Similarly, for lower-middle, higher-middle, and higher level blocks 3, 4, and 5 bit LSB substitution is used. After LSB substitution the pixel values are readjusted to minimize distortion such that these modified values do not disturb the embedded bits. The experimental results reveal that the stego-images are imperceptible and hiding capacity is higher.
\end{abstract}

Keywords: Modified LSB Substitution, Nine-Pixel Differencing, Pixel Value Differencing, Steganography

\section{Introduction}

Steganography is an art of invisible communication, wherein the secret message is sent through a cover medium like image, audio, and video ${ }^{1}$. Digital Image steganography methods are of two categories such as, (i) spatial domain methods, and (ii) frequency domain methods. The image with which the secret message is transmitted is called as the stego-image. By hiding the secret message inside an image, there will be the change in statistics, but this change should be very less such that the intruder will not suspect $i^{2}$. The Least Significant Bit (LSB) substitution method is the simplest and well known image steganographic method. But it is vulnerable to varieties of attacks. So it is strengthened by making some alterations. The LSBs of the various pixels can be grouped together to form an array and the binary secret message can be embedded at a maximum matching portion of it, so that the distortion will be minimum ${ }^{3,4}$. Pharwaha ${ }^{5}$ proposed a Moderate Bit Substitution (MBS) data hiding scheme such that a secret data bit is embedded at a position next to first zero appearing at any of the first three LSB positions in the pixel, but the pixel is avoided if the first three LSBs in it are ' 111 '. This scheme leads to the random selection of bit positions in the given image pixel, so that security is enhanced. If we hide adaptive number of bits in different pixels, then both the security and capacity can be increased ${ }^{6,7}$. Swain and Lenka ${ }^{8-10}$ proposed message bit dependent LSB embedding schemes, wherein the embedding locations in a pixel are randomized depending on the bit pattern of the secret data. For smaller size secret messages only the LSBs of brighter and darker pixels can be targeted, so that embedding can be randomized and security can be improved ${ }^{11,12}$. A pixel in a colored image comprises of 3 bytes. The LSB substitution for colored images can be done differently. One of the bytes can be the indicator to indicate the existence of hidden data in other two bytes ${ }^{13-16}$.

$\mathrm{Wu}$ and $\mathrm{Tsai}^{17}$ proposed Pixel Value Differencing (PVD) steganography with an idea that more number of bits can be embedded in edge areas compared to smooth areas of the image. This scheme is based on the substitution

*Author for correspondence 
of a pixel value difference by a new difference value in every non-overlapping block of two consecutive pixels. Other improved forms of PVD techniques have also been proposed in literature ${ }^{18-20}$. With a slight modification to these original PVD techniques, the side match techniques based on the correlation of a pixel with its surrounding pixels has also been evolved. Chang and Tseng ${ }^{21}$ proposed two-sided, three-sided and four-sided side match methods by exploiting the correlation of a target pixel with its two, three, and four neighboring pixels respectively. PVD techniques with maximum difference of neighboring pixel values have been proposed in ${ }^{22,23}$. The Fall In Error Problem (FIEP) which was observed in Chang and Tseng's method is addressed $\mathrm{in}^{24}$. LSB substitution provides high capacity and less distortion, but not secured. PVD techniques provide high security and more distortion. Wu et al. ${ }^{25}$ proposed a technique with 2-pixel blocks using PVD and 3-bit LSB substitution to achieve high embedding capacity and more security. But it is observed that this technique enforces 3-bit LSB substitution in almost $90 \%$ of the blocks ${ }^{26}$. Furthermore Liao et al. ${ }^{27}$ has proposed a modified LSB substitution in 4-pixel blocks after calculating pixel value differences. But the step- 6 of the embedding procedure i.e. readjusting procedure, searches the new value of the pixels from a large number of possible values, which is not computationally feasible.

Being inspired by Wu et al. ${ }^{25}$ and Liao et al. ${ }^{27}$ schemes and to achieve higher capacity and higher security, a steganographic tehnique with nine-pixel differencing and modified LSB substitution has been proposed in this paper.

In section 2 the proposed technique is described. The results are discussed and compared with the results of $\mathrm{Wu}$ et al's LSB+PVD scheme in section 3. Finally, the paper is concluded in section 4 .

\section{Nine-Pixel Differencing and Modified LSB Substitution Technique}

The image is scanned in raster scan order and is partitioned into non-overlapping blocks consisting $3 \times 3$ pixels as shown in Figure 1, where $\mathrm{x}_{0}, \mathrm{x}_{1}, \mathrm{x}_{2}, \mathrm{x}_{3}, \mathrm{x}_{4}, \mathrm{x}_{5}, \mathrm{x}_{6}, \mathrm{x}_{7}$, and $\mathrm{x}_{8}$ are the different pixel values.

The average difference value, $\mathrm{d}$ of the block is calculated as in equation 1 , where $\mathrm{x}_{\min }$ is the minimum value of $\mathrm{x}_{\mathrm{i}}$, for $\mathrm{i}=0,1,2, \ldots, 8$.

\begin{tabular}{|l|l|l|}
\hline $\mathrm{x}_{0}$ & $\mathrm{x}_{1}$ & $\mathrm{x}_{2}$ \\
\hline $\mathrm{x}_{3}$ & $\mathrm{x}_{4}$ & $\mathrm{x}_{5}$ \\
\hline $\mathrm{x}_{6}$ & $\mathrm{x}_{7}$ & $\mathrm{x}_{8}$ \\
\hline
\end{tabular}

Figure 1. A $3 \times 3$ sample block.

$$
\mathrm{d}=\frac{1}{8} \sum_{\mathrm{i}=0}^{8}\left|\mathrm{x}_{\mathrm{i}}-\mathrm{x}_{\min }\right|
$$

If $\mathrm{d} \leq 7$, then the block belongs to lower-level and 2-bit LSB substitution is applied. If $8 \leq \mathrm{d} \leq 15$, then the block belongs to lower-middle level and 3-bit LSB substitution is applied. If $16 \leq \mathrm{d} \leq 31$, then the block belongs to higher-middle level and 4-bit LSB substitution is applied. If $\mathrm{d} \geq 32$, then the block belongs to higher level and 5-bit LSB substitution is applied.

Suppose n-bit LSB substitution is applied in a block, where $\mathrm{n}$ value is $2,3,4$, and 5 corresponding to lower, lower-middle, higher-middle, and higher level respectively. The two LSBs of $\mathrm{x}_{8}$ i.e. $7^{\text {th }}$ and $8^{\text {th }}$ bit locations are reserved to behave as indicator during extraction, but the other bit locations like $4^{\text {th }}, 5^{\text {th }}$, and $6^{\text {th }}$ can be utilized for data embedding if granted as per the block-level. These $7^{\text {th }}$ and $8^{\text {th }}$ bits are set to 00 if the block belongs to lower level. Similarly, these two bits are set to 01,10 , and 11 if the block belongs to lower-middle, higher-middle, and higher level respectively. Thus a block can hide a total of $(9 \times \mathrm{n}-2)$ number of bits. Let $\mathrm{y}_{0}, \mathrm{y}_{1}, \mathrm{y}_{2}, \mathrm{y}_{3}, \mathrm{y}_{4}, \mathrm{y}_{5}, \mathrm{y}_{6}, \mathrm{y}_{7}$, and $\mathrm{y}_{8}$ are the new pixel values corresponding to $\mathrm{x}_{0}, \mathrm{x}_{1}, \mathrm{x}_{2}, \mathrm{x}_{3}, \mathrm{x}_{4}$, $\mathrm{x}_{5}, \mathrm{x}_{6}, \mathrm{x}_{7}$, and $\mathrm{x}_{8}$ respectively.

After applying the n-bit LSB substitution, now the adjustments are applied to $y_{i}$, for $i=0,1 \ldots 8$, as in equation 2 below, to minimize the distortion. Where $\mathrm{z}_{\mathrm{i}}$, for $\mathrm{i}=0,1 \ldots 8$, modified values.

$$
z_{i}=\left\{\begin{array}{cc}
y_{i}-2^{n}, & \text { if } y_{i} \geq\left(x_{i}+2^{n-1}+1\right. \\
y_{i}+2^{n}, & \text { if } y_{i} \leq\left(x_{i}-2^{n-1}+1\right. \\
y_{i}, & \text { otherwise }
\end{array}\right.
$$

After this adjustment if the $z_{i}$ value falls off boundary $\{0,255\}$, then the equation 3 is applied to enforce it to fall with in boundary $\{0,255\}$.

$$
z_{i}= \begin{cases}z_{i}+2^{n}, & \text { if } z_{i}<0 \\ z_{i}-2^{n}, & \text { if } z_{i}>255\end{cases}
$$


Now, the final stego-block comprises of the pixel values $z_{0}, z_{1}, z_{2}, z_{3}, z_{4}, z_{5}, z_{6}, z_{7}$, and $z_{8}$ as in Figure 2 corresponding to the original pixel block given in Figure 1.

The extraction procedure is very simple. The stegoimage is scanned in raster scan order and is partitioned into non-overlapping blocks consisting $3 \times 3$ pixels as in embedding. Let $\mathrm{s}_{0}, \mathrm{~s}_{1}, \mathrm{~s}_{2}, \mathrm{~s}_{3}, \mathrm{~s}_{4}, \mathrm{~s}_{5}, \mathrm{~s}_{6}, \mathrm{~s}_{7}$, and $\mathrm{s}_{8}$ are the different stego-pixel values and the pixel $s_{8}$ is represented by the eight bits as: $\mathrm{s}_{8}=\mathrm{b}_{1} \mathrm{~b}_{2} \mathrm{~b}_{3} \mathrm{~b}_{4} \mathrm{~b}_{5} \mathrm{~b}_{6} \mathrm{~b}_{7} \mathrm{~b}_{8}$, where each $\mathrm{b}_{\mathrm{i}}$ for $\mathrm{i}=1,2 \ldots 8$ is a bit.

If $b_{7} b_{8}$ is 00 , then two LSBs from each of the pixels $\mathrm{s}_{0}, \mathrm{~s}_{1}, \mathrm{~s}_{2}, \mathrm{~s}_{3}, \mathrm{~s}_{4}, \mathrm{~s}_{5}, \mathrm{~s}_{6}$, and $\mathrm{s}_{7}$ are extracted and from $\mathrm{s}_{8}$ nothing is extracted. If $b_{7} b_{8}$ is 01 , then three LSBs from each of the pixels $s_{0}, s_{1}, s_{2}, s_{3}, s_{4}, s_{5}, s_{6}$, and $s_{7}$ are extracted and from $s_{8}$ the bit from $6^{\text {th }}$ location, i.e. $b_{6}$ is extracted. If $\mathrm{b}_{7} \mathrm{~b}_{8}$ is 10 , then four LSBs from each of the pixels $\mathrm{s}_{0}, \mathrm{~s}_{1}$, $s_{2}, s_{3}, s_{4}, s_{5}, s_{6}$, and $s_{7}$, are extracted and from $s_{8}$ the bits from $5^{\text {th }}$ and $6^{\text {th }}$ locations, i.e. $\mathrm{b}_{5} \mathrm{~b}_{6}$ are also extracted. If $b_{7} b_{8}$ is 11 , then five LSBs from each of the pixels $s_{0}$, $s_{1}, s_{2}, s_{3}, s_{4}, s_{5}, s_{6}$, and $s_{7}$, are extracted and from $s_{8}$ the

\begin{tabular}{|l|l|l|}
\hline$z_{0}$ & $z_{1}$ & $z_{2}$ \\
\hline$z_{3}$ & $z_{4}$ & $z_{5}$ \\
\hline$z_{6}$ & $z_{7}$ & $z_{8}$ \\
\hline
\end{tabular}

Figure 2. The $3 \times 3$ stego-block. bits from $4^{\text {th }}, 5^{\text {th }}$ and $6^{\text {th }}$ locations, i.e. $\mathrm{b}_{4} \mathrm{~b}_{5} \mathrm{~b}_{6}$ are also extracted.

This proposed scheme differs from Liao et al. ${ }^{27}$ scheme in the following points. Firstly, the proposed scheme is based on nine pixel differencing and Liao et al's scheme is based on four pixel differencing. Secondly, the proposed scheme adaptively embeds messages using four levels (lower, lower-middle, higher-middle, and higher), where as Liao et al's scheme uses only two levels (lower and higher). Thirdly, the sixth step in Liao et al's scheme called as "readjustment procedure", searches the new value of the pixels from a large number of possible values, which is not computationally feasible. The readjustment procedure in the proposed scheme is complete different and is as given in equation 2 .

\section{Results and Discussion}

The proposed scheme is implemented using MATLAB. Figure 3 represents a set of original color images of size $256 \times 256$ and Figure 4 represents the corresponding stego-images with 3,50,000 bits of data hidden in each. Similarly, Figure 5 represents a set of original color images of size $512 \times 512$ and Figure 6 represents the corresponding stego-images with $14,000,000$ bits of data hidden in each.

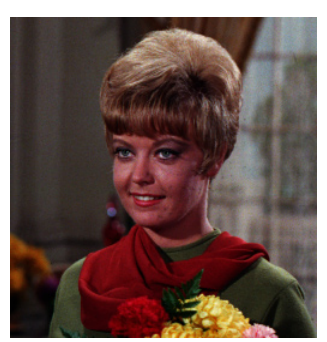

(a) Girl1

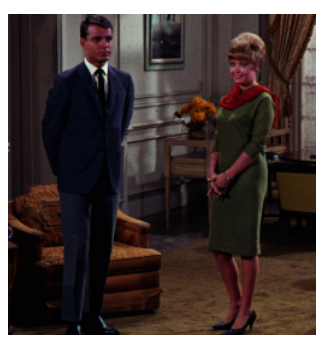

(b) Couple

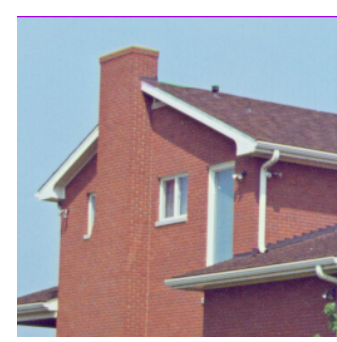

(c) House1

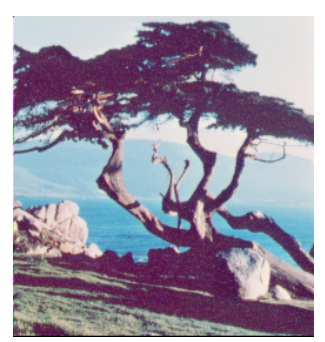

(d) Tree

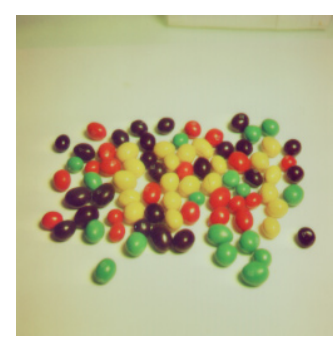

(e) Jelly beans2

Figure 3. Original Images $(256 \times 256)$.

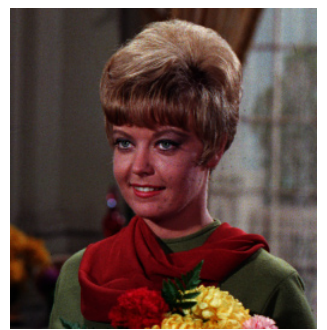

(a) Girl1

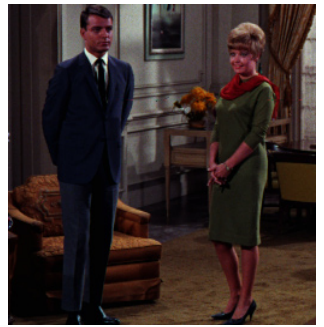

(b) Couple

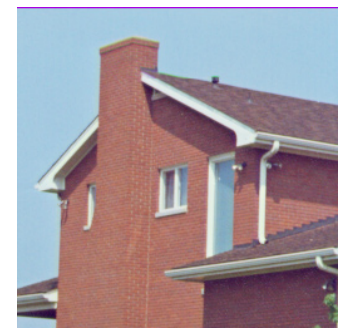

(c) House1

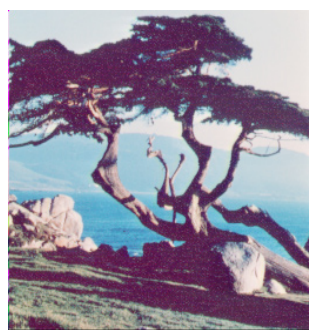

(d) Tree

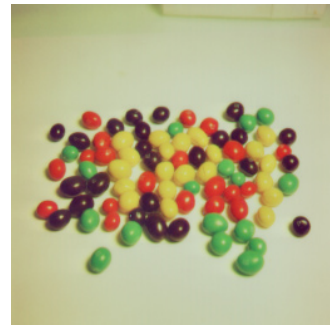

(e) Jelly beans2

Figure 4. Stego-images with 3,50,000 bits of data hidden in each. 


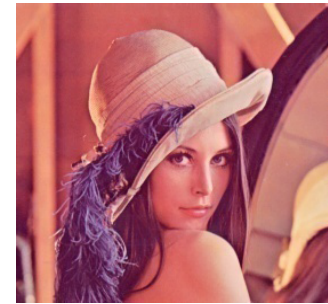

(a) Lena

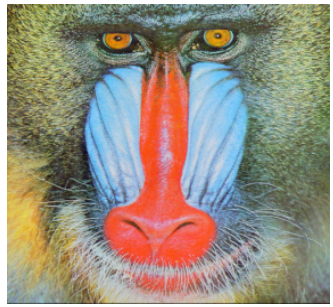

(b) Baboon

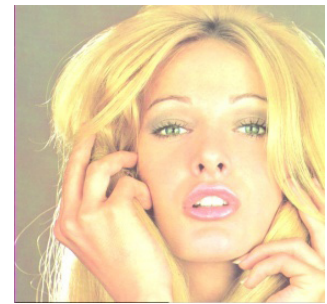

(c) Tiffany

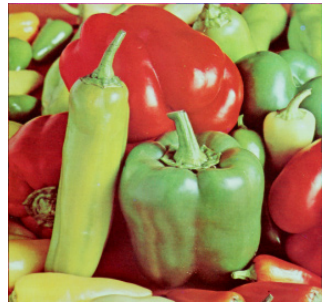

(d) Peppers

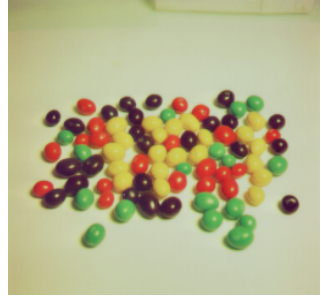

(e) pot

Figure 5. Original Images $(512 \times 512)$.

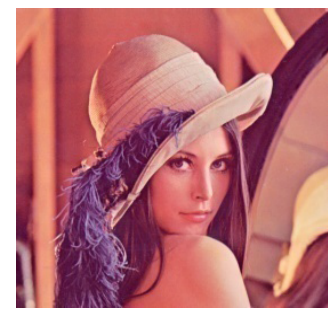

(a) Lena

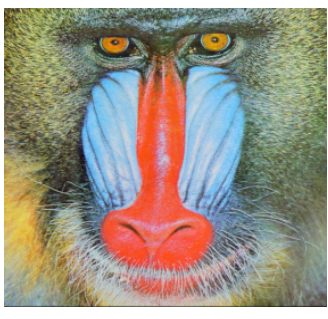

(b) Baboon

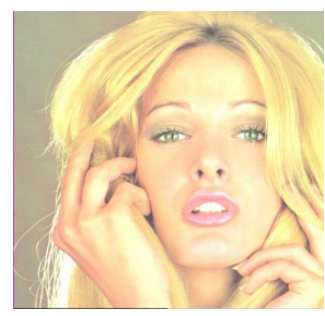

(c) Tiffany

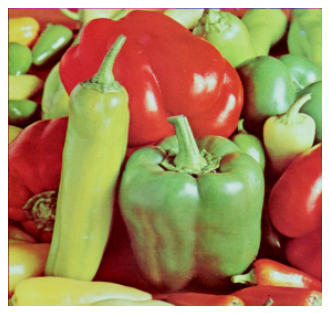

(d) Peppers

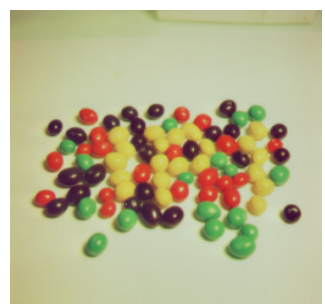

(e) pot

Figure 6. Stego-images with $14,000,000$ bits of data hidden in each.

The performance of the proposed scheme is compared with $\mathrm{Wu}$ et al.s $\mathrm{LSB}+\mathrm{PVD}$ scheme by the parameters, (i) mean square error (MSE), (ii) peak signal-to-noise ratio (PSNR), (iii) correlation, (r), and (iv) capacity. The capacity is measured in bits. A steganography technique should strive for getting higher capacity and lesser distortion. The smaller value of MSE refers to lesser distortion. Unlike MSE the higher PSNR value refers to lesser distortion. The correlation ( $r$ ) is an estimation of similarity between the cover image and its stego-image. The maximum value of $r$ can be 1 , if both cover image and stego-image are the same. Thus a higher value of $r$ implies lesser distortion. The equations to measure MSE, PSNR and correlation are as $\mathrm{in}^{28}$.

Table 1 represents a comparison between Wu et al's scheme with the proposed scheme in terms of MSE, PSNR, $r$, and capacity. The results for ten test images are as shown in this table. It can be observed from Table 1 that the hiding capacity is larger for the proposed scheme in seven images except the Jelly beans2, Tiffany and Pot. The PSNR value in the proposed scheme is higher in nine images except Baboon. So for the majority of the images the proposed scheme performs better than $\mathrm{Wu}$ et al's scheme. Furthermore, by taking the average value of the different comparison parameters the following advantages are observed in the proposed scheme. The MSE decreases by 0.5375 , which is $8.19 \%$. The increase in PSNR is 0.74 , which is $1.83 \%$. The correlation value, $r$ is also increased by 0.0002 and the capacity is increased by 187069 bits, which is $14.66 \%$.

In Table 2 the distribution of PVD and LSB blocks of $\mathrm{Wu}$ et al.'s scheme and the distribution of blocks to various levels in the proposed scheme are presented. It can be observed that in Wu et al.s scheme almost $86.38 \%$ of the blocks are using 3-bit LSB substitution. In the proposed scheme only $74.74 \%$ of the blocks are using 3 -bit LSB substitution. The rest $25.26 \%$ of the blocks are using 2-bit, 4-bit and 5-bit LSB substitution. By scattering the distribution of the blocks to these four levels and embedding variable number of bits in the different pixels, the security aspect has been addressed.

It can also be observed from Figure 4 and Figure 6 that the stego-images are of good quality, they do not show any visible marks to be suspected and they are very much similar to their respective original images in Figure 3 and Figure 5.

The proposed scheme can escape from RS steganalysis. In the traditional 1-bit LSB substitution steganography if the LSB of cover image pixel value is equal to the message bit, the pixel value is not altered. Otherwise, the pixel value is altered from $2 n$ to $2 n+1$ or from $2 n+1$ to $2 n$. But the alterations from $2 n$ to $2 n-1$ or $2 n+1$ to $2 n+2$ do not occur. This asymmetry could be caught by RS steganalysis. In the proposed scheme n-bit LSB substitution is applied in a block, where $\mathrm{n}$ value is $2,3,4$, and 5 corresponding to lower, lower-middle, higher-middle, and 
Table 1. Comparison of MSE, PSNR, $r$ and Capacity

\begin{tabular}{|c|c|c|c|c|c|c|c|c|}
\hline \multirow[t]{2}{*}{ Images } & \multicolumn{4}{|c|}{ Wu et al.s LSB + PVD scheme } & \multicolumn{4}{|c|}{ Proposed scheme } \\
\hline & MSE & $\begin{array}{c}\text { PSNR } \\
(\mathrm{dB})\end{array}$ & $\mathbf{r}$ & $\begin{array}{c}\text { Capacity } \\
\text { (bits) }\end{array}$ & MSE & $\begin{array}{c}\text { PSNR } \\
(\mathrm{dB})\end{array}$ & $\mathbf{r}$ & $\begin{array}{c}\text { Capacity } \\
\text { (bits) }\end{array}$ \\
\hline Girl1 & 3.8995 & 42.22 & 0.9988 & 534994 & 2.9854 & 43.38 & 0.9991 & 569929 \\
\hline Couple & 4.8820 & 41.24 & 0.9976 & 532073 & 3.9785 & 42.13 & 0.9980 & 553882 \\
\hline House1 & 3.4040 & 42.81 & 0.9991 & 563833 & 3.2775 & 42.97 & 0.9992 & 571801 \\
\hline Tree & 4.6102 & 41.49 & 0.9994 & 468664 & 4.4748 & 41.62 & 0.9994 & 626638 \\
\hline Jelly beans 2 & 3.1627 & 43.13 & 0.9991 & 554193 & 2.1138 & 44.88 & 0.9994 & 520483 \\
\hline Lena & 7.6717 & 39.28 & 0.9979 & 2111731 & 5.6115 & 40.64 & 0.9984 & 2297680 \\
\hline Baboon & 14.9552 & 36.38 & 0.9974 & 1449956 & 19.5127 & 35.22 & 0.9966 & 2877658 \\
\hline Tiffany & 7.5496 & 39.35 & 0.9956 & 2167738 & 6.2199 & 40.19 & 0.9965 & 2159377 \\
\hline Peppers & 8.1572 & 39.01 & 0.9984 & 2095811 & 7.2495 & 39.52 & 0.9986 & 2286574 \\
\hline Pot & 7.3374 & 39.47 & 0.9987 & 2281844 & 4.8308 & 41.29 & 0.9991 & 2167504 \\
\hline Average & 6.5629 & 40.44 & 0.9982 & 1276083 & 6.0254 & 41.18 & 0.9984 & 1463152 \\
\hline Improvement & & & & & -0.5375 & 0.74 & 0.0002 & 187069 \\
\hline
\end{tabular}

Table 2. Comparison of range counts

\begin{tabular}{|c|c|c|c|c|c|c|}
\hline \multirow[t]{2}{*}{ Images } & \multicolumn{2}{|c|}{ Wu et al.s scheme } & \multicolumn{4}{|c|}{ Proposed scheme } \\
\hline & LSB count & PVD count & $\begin{array}{c}\text { Lower-level } \\
\text { count }\end{array}$ & $\begin{array}{l}\text { Lower-middle } \\
\text { level count }\end{array}$ & $\begin{array}{l}\text { Higher-middle } \\
\text { level count }\end{array}$ & $\begin{array}{l}\text { Higher-level } \\
\text { count }\end{array}$ \\
\hline Girl1 & 88791 & 9513 & 761 & 18102 & 2152 & 745 \\
\hline Couple & 88645 & 9659 & 2339 & 16690 & 2025 & 706 \\
\hline House1 & 92161 & 6143 & 1097 & 17807 & 1526 & 1330 \\
\hline Tree & 78110 & 20194 & 1147 & 13762 & 3373 & 3478 \\
\hline Jelly beans2 & 91444 & 6860 & 6498 & 12683 & 1273 & 1306 \\
\hline Lena & 351487 & 41729 & 353 & 75857 & 7787 & 3043 \\
\hline Baboon & 241342 & 151874 & 12 & 34124 & 27834 & 25070 \\
\hline Tiffany & 361289 & 31927 & 12428 & 66191 & 6261 & 2160 \\
\hline Peppers & 349278 & 43938 & 2146 & 74204 & 6948 & 3742 \\
\hline Pot & 380305 & 12911 & 6229 & 77129 & 2079 & 1603 \\
\hline \multirow[t]{3}{*}{ Average } & 212285 & 33474 & 3301 & 40654 & 6125 & 4318 \\
\hline & $86.38 \%$ & $13.62 \%$ & $6.07 \%$ & $74.74 \%$ & $11.26 \%$ & $7.93 \%$ \\
\hline & \multicolumn{2}{|c|}{$212285+33474=245759(100 \%)$} & \multicolumn{3}{|c|}{$3301+40654+6125+4318=54398(100 \%)$} & \\
\hline
\end{tabular}

higher level respectively. In case of 2-bit LSB substitution if the 2 LSBs of cover image pixel value are equal to the two message bits, the pixel value is not altered. Otherwise the pixel value alters from $2 n$ to $\{2 n+1$ or $2 n-1$ or $2 n-2\}$, or $2 n+1$ to $\{2 n$ or $2 n-1$ or $2 n-2\}$ or $2 n-1$ to $\{2 n$ or $2 n$ +1 or $2 n-2\}$, or $2 n-2$ to $\{2 n$ or $2 n+1$ or $2 n-1\}$. Note that a pixel value is considered in one of these four ways like $\{2 n, 2 n+1,2 n-1,2 n-2\}$, where $n=1,2, \ldots, 127$. Thus there is no asymmetry as arises in 1-bit LSB substitution. Similarly in 3-bit LSB, 4-bit LSB, and 5-bit LSB substitution also this asymmetry does not occur. From this discussion it is clear that the proposed scheme can escape from RS steganalysis. 


\section{Conclusion}

To achieve higher embedding capacity and lesser distortion an adaptive and modified LSB substitution steganographic scheme has been proposed. The security has been addressed by hiding variable number of bits in different blocks by categorizing the blocks into one of the four levels (lower, lower-middle, higher-middle, and higher) based on average of pixel value differences in nine-pixel blocks. In a majority of the test cases the capacity of the proposed scheme is higher and the distortion is lower as compared to the Wu et al's LSB + PVD scheme. The stego-images are imperceptible and do not show any visible marks to draw the attention of the intruders. The extraction can be done very simply by using the two LSBs of the $9^{\text {th }}$ pixel of every $3 \times 3$ block.

\section{References}

1. Cheddad A, Condell J, Curran K, Kevitt PM. Digital image steganography: survey and analysis of current methods. Signal Processing. 2010; 90:727-52.

2. Martin A, Sapiro G, Seroussi G. Is image steganography natural?. IEEE Trans Image Process. 2005; 14(12):2040-50.

3. Juneja M, Sandhu PS. Designing of robust image steganography technique based on LSB insertion and encryption. International Conference on Advances in Recent Technologies in Communication and Computing; 2009 Oct 27-28; Kottayam, Kerala. IEEE; 2009 Nov. p.302-5.

4. Swain G, Lenka SK. LSB array based image steganography technique by exploring the four least significant bits. CCIS. 2012; 270(2):479-88.

5. Pharwaha APS. Secure data communication using moderate bit substitution for data hiding with three layer security. IE(I) Journal-ET. 2010; 91:45-50.

6. He J, Tang S, Wu T. An adaptive steganography based on depth-varying embedding. Congress on Image and Signal Processing. 2008 May 27-28; Sanya, China. IEEE; 2008. p.660-3.

7. Jain YK, Ahirwal RR. A novel image steganography method with adaptive number of least significant bits modification based on private stego-keys. IJCSS. 2010; 4(1):40-9.

8. Swain G, Lenka SK. A robust image steganography technique using dynamic embedding with two least significant bits. Adv Mater Res. 2012; 403-408:835-41.

9. Swain G, Lenka SK. A dynamic approach to image steganography using the three least significant bits and extended hill cipher. Adv Mater Res. 2012; 403-408:842-9.

10. Swain G, Lenka SK. A technique for secret communication by using a new block cipher with dynamic steganography.
International Journal of Security and Its Applications. 2012; 6(2):1-12.

11. Swain G, Lenka SK. A hybrid approach to steganographyembedding at darkest and brightest pixels. International Conference on Communication and Computational Intelligence; 2010 Dec 27-29; Erode. IEEE; 2010. p.529-34.

12. Swain G, Lenka SK. Application of a large key cipher in image steganography by exploring the darkest and brightest pixels. Int J Comput Sci Comm. 2012; 3(1):49-53.

13. Parvez MT, Gutub AA. RGB based variable-bits image steganography. IEEE Asia Pacific Services Computing Conference; 2008 Dec 9-12; Yilan. IEEE; 2008. p.1322-7.

14. Tiwari N, Shandilya M. Secure RGB image steganography from pixel indicator to triple algorithm- an incremental growth. International Journal of Security and Its Applications. 2010; 4(4):53-62.

15. Swain G, Lenka SK. A better RGB channel based image steganography technique. CCIS. 2012; 270(2):470-8.

16. Swain G, Lenka SK. A novel approach to RGB channel based image steganography technique. International Arab Journal of e-Technology. 2012; 2(4):181-6.

17. Wu DC, Tsai WH. A Steganographic method for images by pixel-value differencing. Pattern Recogn Lett. 2003; 24:1613-26.

18. Zhang X, Wang S. Vulnerability of pixel-value differencing steganography to histogram analysis and modification for enhanced security. Pattern Recogn Lett. 2004; 25:331-9.

19. Chang KC, Chang CP, Huang PS, Tu TM. A novel image steganographic method using tri-way pixel-value differencing. Journal of Multimedia. 2008; 3(2):37-44.

20. Lee YP, Lee JC, Chen WK, Chang KC, Su IJ, Chang CP. High-payload image hiding with quality recovery using tri-way pixel-value differencing. Inform Sci. 2012; 191:214-25.

21. Chang CC, Tseng HW. A steganographic method for digital images using side match. Pattern Recogn Lett. 2004; 25:1431-7.

22. Pradhan A, Sharma DS, Swain G. Variable rate steganography in digital images using two, three and four neighbor pixels. Indian Journal of Computer Science and Engineering. 2012; 3(3):457-63.

23. Swain G. Steganography in digital images using maximum difference of neighboring pixel values. International Journal of Security and Its Applications. 2013; 7(6):285-94.

24. Swain G, Lenka SK. Steganography using two sided, three sided and four sided side match methods. CSI Transactions on ICT. 2013; 1(2):127-33.

25. Wu HC, Wu NI, Tsai CS, Hwang MS. Image steganographic scheme based on pixel-value differencing and LSB replacement methods. IEEE Proceedings-Vision, Image and Signal Processing. 2005; 152(5):611-5. 
26. Yang CH, Weng CY, Wang SJ, Sun HM. Varied PVD + LSB evading programs to spatial domain in data embedding systems. J Syst Software. 2010; 83:1635-43.

27. Liao X, Wen QY, Zhang J. A steganographic method for digital images with four-pixel differencing and modified
LSB Substitution. Journal of Visual Communication and Image Representation. 2011; 22:1-8.

28. Swain G, Lenka SK. Classification image steganography techniques in spatial domain: A study. Int J Comput Sci Eng Tech. 2014; 5(3):219-32. 